\title{
A parametric study of laser interference surface patterning of dental zirconia: effects of laser parameters on topography and surface quality
}

\author{
E. Roitero $^{1,2,3, *}$, F. Lasserre ${ }^{3}$, M. Anglada ${ }^{1,2}$, F. Mücklich ${ }^{3}$ and E. Jiménez-Piqué 1,2 $^{12}$
}

\footnotetext{
${ }^{1}$ Department of Materials Science and Metallurgical Engineering, Universitat Politècnica de Catalunya, Av. Diagonal 647, Barcelona 08028, Spain.

${ }^{2}$ Centre for Research in Nanoengineering, CRnE, Univerisitat Politèncica de Catalunya, C. Pascual i Vila 15, Barcelona 08028, Spain.

${ }^{3}$ Department of Materials Science and Engineering, Saarland University, Saarbrucken 66123, Germany.

*Corresponding Author

E-mail: erica.roitero@upc.edu
}

\section{ABSTRACT}

Objectives. The aim of this work is to generate micrometric linear patterns with different topography on dental grade zirconia by means of UV laser interference and to assess the quality of the produced surface, both in term of the geometry produced and of the surface damage induced in the material.

Methods. The third harmonic of a Q-switched Nd:YAG laser (355 nm, pulse duration of $10 \mathrm{~ns}$ and repetition rate of $1 \mathrm{~Hz}$ ) was employed to pattern the surface of 3Y-TZP with micrometric-spaced lines. The resulting topography was characterized with White Light Interferometry and Scanning electron microscopy: pattern depth $(H)$, amplitude roughness parameters $\left(S_{a}\right.$, filtered- $\left.S_{a}\right)$, Fourier spatial analysis and collateral damages were related to laser fluence and number of pulses employed.

Results. With our experimental setup, line-patterning of zirconia surfaces can be achieved with periodicities comprised within 5 and $15 \mu \mathrm{m}$. Tuning laser parameters allows varying independently pattern depth, overall roughness and surface finish. Increasing both fluence and number of pulses allows producing deeper patterns (maximum achievable depth of $1 \mu \mathrm{m}$ ). However, increasing the number of pulses has a detrimental effect on the quality of the produced lines. Surface damage (intergranular cracking, open porosity and nano-droplets formation) can be generated, depending on laser parameters.

Significance. This work provides a parametric analysis of surface patterning by laser interference on 3YTZP. Best conditions in terms of quality of the produced pattern and minimum material damage is obtained for low number of pulses with high laser fluence. With the employed method we can produce zirconia materials with controlled topography that are expected to enhance biological response and mechanical performance of dental components.

Keywords: zirconia, 3Y-TZP, laser, surface patterning. 


\section{INTRODUCTION}

Ceramic materials are commonly used for dental applications including veneering material for metal substructures, all-ceramic posts and cores, frameworks for crowns and bridge-works [1]. At the moment, there is an increasing interest in using ceramics also as materials for oral implants [2] as an alternative to titanium due to their higher resistance to bio-corrosion and superior aesthetic properties $[3,4]$. Tetragonal Zirconia Polycrystal stabilized with $3 \%$ molar of Yttrium oxide (3Y-TZP) is considered an excellent material for all these applications because of its bio-stability, mechanical properties (good strength, hardness and high fracture toughness) and aesthetic appearance in combination with porcelain enamel (white ivory color, close to natural teeth) [4,5]. Furthermore, it has been demonstrated that zirconia ceramic exhibits low plaque accumulation and displays similar bacterial binding properties to titanium [6].

Despite the aforementioned advantages, the suitability of 3Y-TZP to be used in the dental prosthetic field depends on the feasibility of functionalizing its surface to improve the biological response or the mechanical adhesion to other materials (like dental cements and resins) [7]. Of course, this should be carried out by techniques that do not compromise material's bulk properties and long-term stability. Common strategies to achieve these goals are the modification of the roughness or the introduction of a controlled topography. For instance, micrometric silica reliefs on zirconia implant surfaces have been employed to study endothelial cells and fibroblast response in [8], where it was demonstrated that parallel grooved surfaces were able to control cells spatial distribution and to guide their growth. Also an increment in roughness has proved to be beneficial in the osseointegration of zirconia-based implants [9]. On the other hand, the adhesion of dental cement to roughed surfaces of Zirconia have been improved both with grinding[10] or laser machining[11,12]. [u1]

The most common physical surface modification methods available for ceramics are machine-aided approaches such as sandblasting[13], grinding[14], acid etching[15] or laser micromachining[16,17]. The last rises above the others as suggested by M. Holthaus et al.[18] in a comparative study of different techniques to pattern the surface of ceramics materials such as alumina, zirconia, silica and 
hydroxyapatite. They concluded that laser treatment processes are a suitable alternative to classical methods since they have the advantage of being fast and with a high control on the final desired topography in contrast with contact techniques, as mechanical micromachining or stamp transfer molding. Moreover, using conventional methods the fabrication of defined patterns smaller than $100 \mu \mathrm{m}$ is still challenging due to the high hardness and brittleness[19] of ceramics.

Direct Laser Interference Patterning (DLIP) offers a fast and accurate alternative to introduce controlled topography at the micrometric and sub-micrometric scale[19]. In this technique a periodical intensity distribution is produced by beam interference on the surface of the material to be treated. Depending on the number of interfering laser beams and the optical setup, different geometries can be produced (lines or dots). Commonly, nanosecond, picosecond and femtosecond pulsed lasers are used in order to reach high energy density at the interference maxima position. This high peak power permit to locally melt, vaporize or ablate the substrate to engrave the desired geometry[20]. Further details about the technique and the achievable patterns can be found in [21]. DLIP allows great precision and flexibility in the produced topography and is fast enough to modify large areas, especially if compared to other laser micromachining techniques that require the scanning of the beam through the surface by optomechanical methods[22]. DLIP has been successfully used to pattern surfaces of different materials: from metals[23-26] to ceramics[27-29] and polymers[30,31]. Most common applications are in the field of tribology and biomaterials: topographical, chemical and microstructural modifications induced by DLIP are exploited to modify surface wettability[23], to introduce texture[25] or to tune the interaction with biological species[30,31].

DLIP technique has been successfully applied to produce micrometric line-patterning onto 3Y-TZP with neither significant collateral damage induced by the laser treatment [28] nor detrimental effect on mechanical properties [32]. However, there has not been any systematic study about the influence of laser parameters on the topographies produced with DLIP on 3Y-TZP. As demonstrated for other ceramic materials, changing laser parameters employed often results in different surface topographies 
[27]. Moreover, a detailed roughness analysis at different scales is essential to accurately describe the topography of the produced patterns, especially when dealing with biomedical applications [33]

The objective of this work is to correlate the morphology and the quality of the generated pattern to laser parameters employed (fluence and number of pulses). The topography and roughness of the patterns are characterized by means of $2 \mathrm{D}$ - and $3 \mathrm{D}$-amplitude parameters and spatial distribution analysis. Surface finish and quality are assessed in terms of collateral damages induced by laser treatment. Finally, the processability ranges are determined as a function of laser parameters.

\section{EXPERIMENTAL}

\subsection{Material processing}

Commercially available powder of Tetragonal Polycrystalline Zirconia stabilized with $3 \%$ molar $\mathrm{Y}_{2} \mathrm{O}_{3}$ (TZ3YSB-E, Tosoh Co.) was employed. The powder was isostatically pressed at $200 \mathrm{MPa}$ in a cylindrical mold and subsequently sintered in an alumina tube furnace at $1450{ }_{-}^{\circ} \mathrm{C}$ for two hours (heating rate: $3{ }^{\circ} \mathrm{C} / \mathrm{min}$ ), as described in previous work [34]. The resulting rods (10 $\mathrm{mm}$ in diameter) were cut into discs of approximately $2 \mathrm{~mm}$ thickness. The surface of the discs were grinded and polished with diamond suspensions of decreasing particle size (30 - $6-3 \mu \mathrm{m})$ and with colloidal silica as a final step. The samples had a final density of $6.03 \pm 0.02 \mathrm{~g} / \mathrm{cm}^{3}$ (99.67\% of theoretical density[F2]) and a grain size (intercept distance) of $0.31 \pm 0.08 \mu \mathrm{m}$. The obtained material has biomedical grade, according to ISO 13356:2013 [35].

\subsection{Laser treatment}

A Q-switched Nd:YAG laser (Spectra Physics Quanta-Ray PRO210) with a fundamental wavelength of 1064 nm and an output wavelength of 355 nm obtained by $3^{\text {rd }}$ harmonic generation was employed to pattern the surface of zirconia discs. The repetition rate and pulse duration of this laser were $10 \mathrm{~Hz}$ and $10 \mathrm{~ns}$, respectively. A mechanical shutter (nmLaser LSTXY-W8) was used to isolate pulses and reduce 
frequency to $1 \mathrm{~Hz}$. The optical setup employed to produce the DLIP is illustrated in Figure 1. Further details about the technique and the setup employed can be found in [26] and [24].

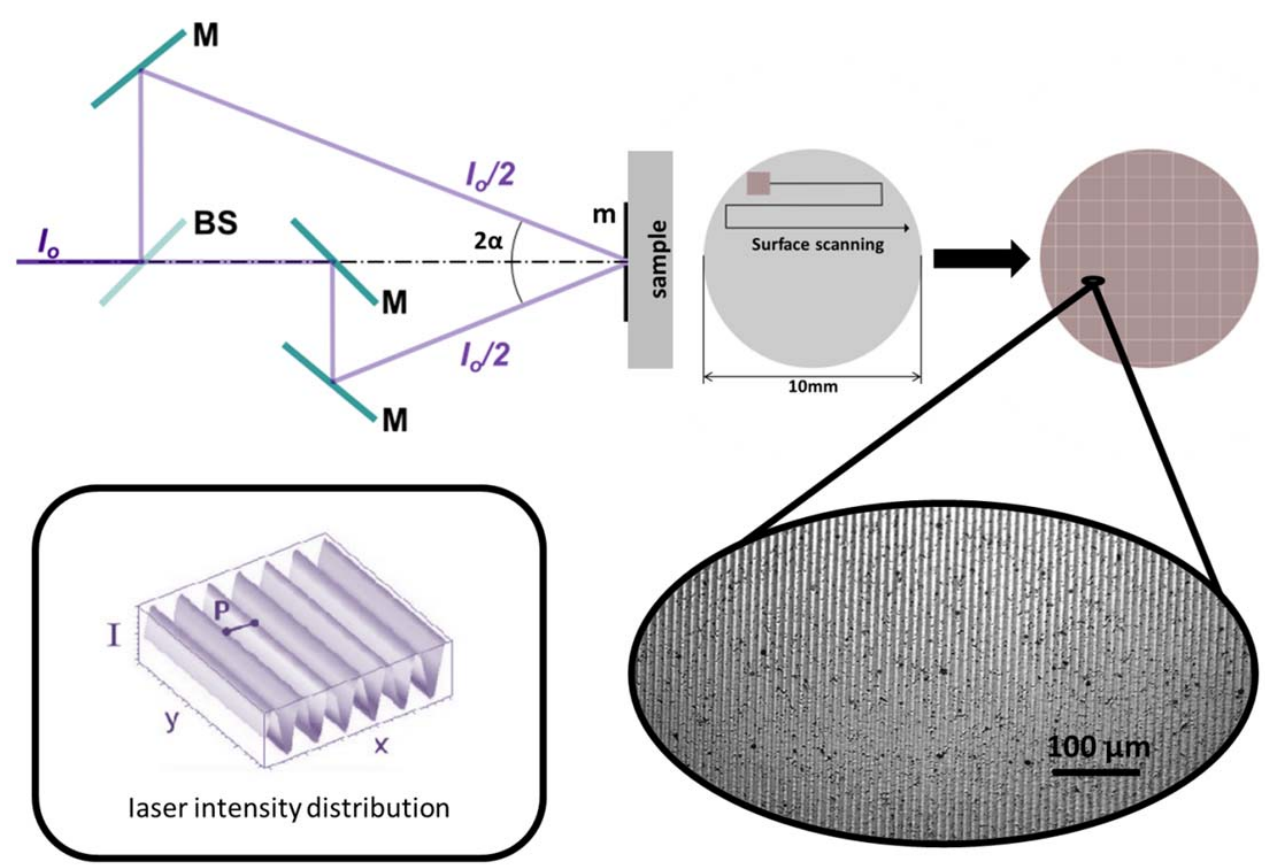

Figure 1. Optical setup for Direct Laser Interferce patterning (DLIP) with two beams configuration to produce a linear pattern. The incoming laser beam is split into two at the beam splitter (BS) and the two secondary beams are redirected by a mirror $(\mathrm{M})$ setup towards the surface where interference is produced. A squared mask $(m)$ is placed before the sample surface so that the entire surface can be patterned with subsequent adjacent spots. In the inset, the laser intensity distribution along the sample and the periodicity $(P)$ are depicted. At the bottom right, a linear pattern produced on a sample surface is shown.

By the use of a beam spliter two coherent sub-beams are generated from a primary one. Mirrors guide the two beams to the sample surface, where they are left interfere. The resulting intensity distribution is a plane sinusoidal and can be mathematically described by: 
$I(x, y)=I_{o}\left[\cos \left(\frac{4 \pi x}{\lambda} \sin \alpha\right)+1\right]$

where $I_{0}$ is the intensity of the laser beam before splitting, $\lambda$ is the laser wavelength and $\alpha$ is the half angle between the interfering beams. The same relation can be expressed in terms of fluence $(F(x, y))$, which then represents the laser energy density distribution on the surface of the sample. The two quantities are directly related since the fluence is defined as the pulse energy per unit area and the intensity is defined as the peak power (i.e. the pulse energy divided by the pulse duration) per unit area[36]. The fluence distribution consists of alternating lines of energy maximum $\left(F_{\max }=2 F_{0}\right)$ and minimum $\left(F_{\min }=0\right)$. From now on in the paper we will refer to $F_{o}$ as "fluence".

The line-pattern then has a periodicity $(P)$ of:

$P=\frac{\lambda}{2 \sin \alpha}$

Varying the incidence angle of the two beams, different line-periodicities were tested (1-4-7-10$15 \mu \mathrm{m}$ ). A beam attenuator (Altechna UAB) and a mechanical shutter (nmLaser LSTXY-W8) were employed to vary the power density of the beam and the number of pulses reaching the surface of the sample. A square mask of $1 \times 1 \mathrm{~mm}^{2}$ placed before the sample allowed the selection of a homogeneous portion of the incoming beam for patterning the entire surface of the discs with multiple adjacent spots. The beam power was measured with a power detector (Gentec-EO UP19-H) both after the beam attenuator and after the mask, in order to precisely take into account energy losses within the components of the optical setup. The influence of laser fluence and pulse number was investigated in the range of $0.15-7.15 \mathrm{~J} / \mathrm{cm}^{2}$ and $1-10$ pulses, respectively.

\subsection{Topography characterization as a function of laser parameters}

A preliminary analysis was done to determine the range of achievable periodicities and to choose the optimal one to characterize in detail the influence of laser parameters on pattern morphology. The 
surface of laser-treated samples was characterized with White Light Interferometry (WLI, Veeco Wyko 9300NT) in order to verify if the striped pattern was formed. WLI Images of $250 \times 250 \mu \mathrm{m}^{2}$ were obtained by stitching of nine images taken with 50x magnification (total resolution of $1263 \times 1263$ pixels). To assess the effect of laser parameters on the morphology of the treated surfaces, a detailed topographical characterization was carried out on samples with constant line periodicity of $10 \mu \mathrm{m}$ (the reason for this is later discussed). In order to separate the effect of fluence and number of pulses, each series of samples treated with $n$ pulses was exposed to increasing fluence (see Table 1 for the list of conditions employed).

\begin{tabular}{|c|c|c|c|c|c|c|c|c|c|c|c|c|}
\hline & \multicolumn{12}{|c|}{ Fluence per pulse $\left[\mathrm{J} / \mathrm{cm}^{2}\right]$} \\
\hline & 0.15 & 0.20 & 0.25 & 0.38 & 0.63 & 1.13 & 2.15 & 3.15 & 4.15 & 5.15 & 6.15 & 7.15 \\
\hline & 0.15 & 0.20 & 0.25 & 0.38 & 0.63 & 1.13 & 2.15 & 3.15 & 4.15 & 5.15 & 6.15 & 7.15 \\
\hline & 0.30 & 0.40 & 0.50 & 0.77 & 1.27 & 2.27 & 4.30 & 6.30 & 8.30 & 10.30 & 12.30 & 14.30 \\
\hline & 0.75 & 1.00 & 1.25 & 1.92 & 3.17 & 5.67 & 10.75 & 15.75 & 20.75 & 25.75 & 30.75 & 35.75 \\
\hline 10 & 1.50 & 2.00 & 2.50 & 3.83 & 6.34 & 11.35 & 21.50 & 31.50 & 41.50 & 51.50 & 61.50 & 71.50 \\
\hline
\end{tabular}

Table 1. Total fluence $\left[\mathrm{J} / \mathrm{cm}^{2}\right]$ on the sample as a function of number of pulses and fluence per pulse

In order to describe the quality of the achieved pattern, significant roughness parameters were selected (see Table 2 for definition and description). To evaluate those, WLI Images of $633 \times 474 \mu m^{2}(10 x$ magnification and resolution of $640 \times 480$ pixels) and of $127 \times 96 \mu \mathrm{m}^{2}$ (50x magnification and resolution of $640 \times 480$ pixels) were acquired. Height maps and profiles were treated with commercially available software and surface tilt was always corrected. For pattern depth $(H)$ and average roughness $\left(S_{a}\right)$ calculation no filtering was applied in order to evaluate the real depth of the structure and take into account the contribution of all scales features, as it has already been done in a similar study[27]. In a second stage, a Robust Gaussian filter (high-frequency pass with cut-off length of $2.5 \mu \mathrm{m}$ ) was employed to separate the pattern produced with DLIP from smaller size features $\left(f-S_{a}\right)$. Finally, power spectral density (PSD), a Fourier spatial parameter, was employed to describe the neatness of the stripes 
produced. The chosen roughness parameters were calculated for all the conditions and plotted as a function of laser fluence and number of pulses.

\begin{tabular}{|c|c|c|c|}
\hline parameter & symbol & category & definition and description \\
\hline Pattern Height & $H$ & Amplitude (2D) & $\begin{array}{l}\text { Average of the height difference between consecutive peak and } \\
\text { valley of the pattern, calculated on profiles orthogonal to the lines }\end{array}$ \\
\hline 3D Average Roughness & $S_{a}$ & Amplitude (3D) & Average of height values calculated over the entire surface \\
\hline Filtered 3D Average Roughness & $f-S_{a}$ & Amplitude (3D) & $\begin{array}{l}\text { Average of height values calculated over the entire surface after a } \\
\text { high pass filter is applied }\end{array}$ \\
\hline Power Spectral Density & PSD & Spatial & $\begin{array}{l}\text { Distribution of spatial frequencies, calculated on profiles orthogonal } \\
\text { to the lines }\end{array}$ \\
\hline
\end{tabular}

topography

Afterward, Scanning Electron Microscopy (SEM) (Neon 40, Carl Zeiss) images allowed a closer inspection

of the state of the surfaces, i.e. the damage associated to the laser treatment was identified and described as a function of laser parameters.

\section{RESULTS}

Figure 2 presents the surface topographies obtained by DLIP, where the linear pattern can be clearly seen. Its period, depth and quality depend on the laser parameters and setup used. 

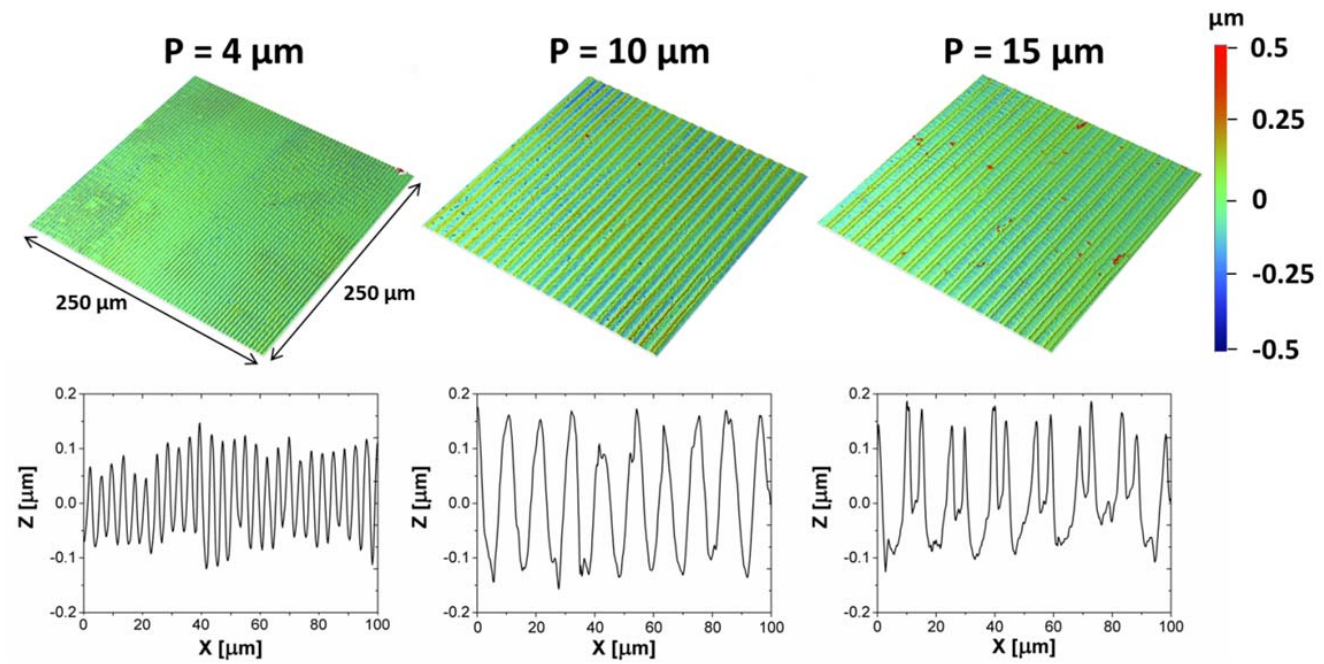

Figure 2. WLI images of DLIP treated zirconia surfaces with different line-periodicities $(P)$, and profiles extracted orthogonally to pattern lines. Samples were treated with one pulse and a fluence of $4 \mathrm{~J} / \mathrm{cm}^{2}(P$ $=4 \mu \mathrm{m})$ and $7 \mathrm{~J} / \mathrm{cm}^{2}(P=10 \mu \mathrm{m}$ and $P=15 \mu \mathrm{m})$.

The pattern consists of an alternation of peaks and valleys with regular periodicity and sinusoid-like shape. A valley corresponds to laser fluence maximum while a peak corresponds to a minimum. Varying the incidence angle of the laser setup, striped patterns of $4-10-15 \mu \mathrm{m}$ periodicities were produced on the surface of 3Y-TZP (Fig. 2). For 4 and $10 \mu \mathrm{m}$ periodicity pattern shape is similar: the profile is almost sinusoidal with a peak to peak distance equal to the desired periodicity. For $P=15 \mu \mathrm{m}$ a double peak is formed, instead, introducing a second type of periodicity to the pattern and leaving an intermediate band of untreated material in between. Patterns with periodicity below $1 \mu \mathrm{m}$ could not be produced with enough precision since the treated surface had a homogeneously molten appearance. Patterns with periods larger than $15 \mu \mathrm{m}$ could be achieved, but comprised an important intermediate area of untreated substrate between two adjacent peaks. It was therefore decided to perform the parametric study at a constant periodicity of $10 \mu \mathrm{m}$ because this intermediate value allowed tuning laser fluence and pulse number in a relatively large interval inducing comparable patterns with different depth, roughness and neatness. 


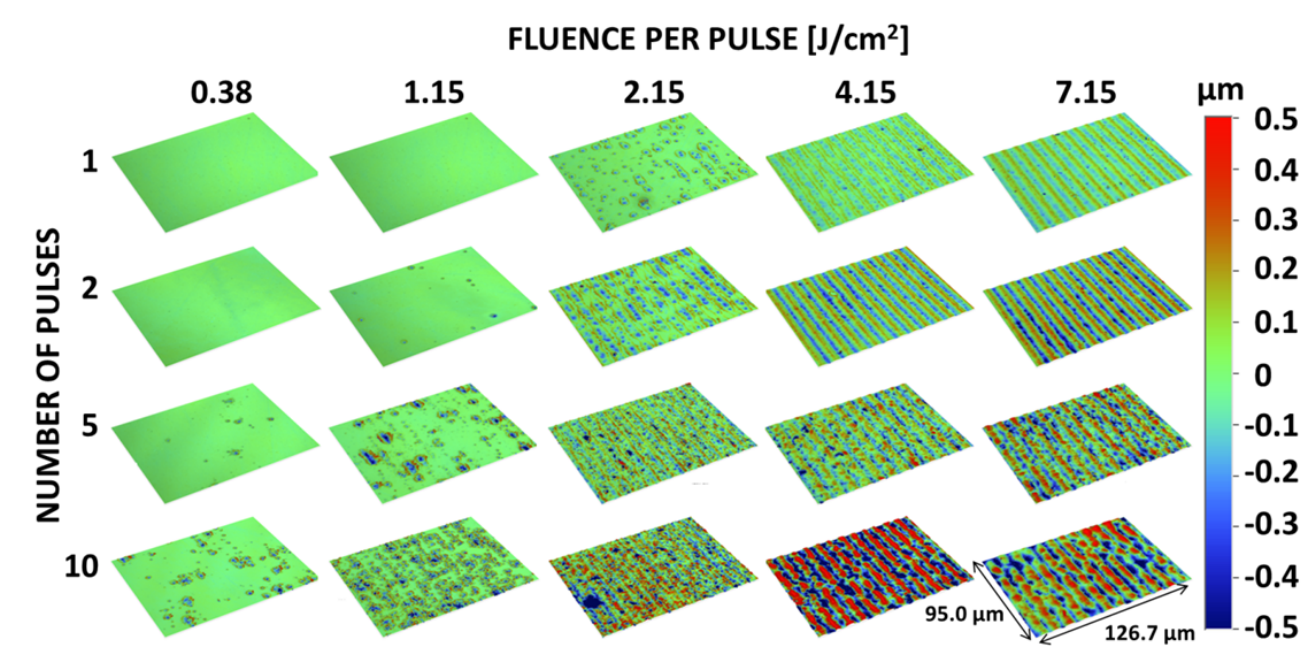

Figure 3. WLI images of DLIP treated zirconia surfaces with different laser parameter combinations.

Pattern evolution as a function of increasing fluence and increasing number of pulses. All samples has a periodicity of $10 \mu \mathrm{m}$.

Figure 3 shows the 3D topography of the $10 \mu \mathrm{m}$ periodical structures obtained after laser treatments at different laser conditions. It is worth noting that it is possible to produce a pattern even with one single pulse: for low fluences (between $2.15 \mathrm{~J} / \mathrm{cm}^{2}$ and $4.15 \mathrm{~J} / \mathrm{cm}^{2}$ ) the engraved lines do not form completely while for higher values (above $4.15 \mathrm{~J} / \mathrm{cm}^{2}$ ) they are completely developed. This trend is similar when patterning with more pulses. However, for small number of pulses (i.e. one or two) the pattern is more homogeneous and neat while for treatments with higher number of pulses (i.e. five or ten) the structure becomes more irregular and lines are less defined.

A fluence threshold can be identified as the minimum fluence at which material modification or removal can be observed on the surface [36] and it decreases with increasing number of pulses, as presented in table 3.

1

2

5

10


Table 3. Threshold fluence values obtained for $1,2,5$ and 10 pulses treatments.
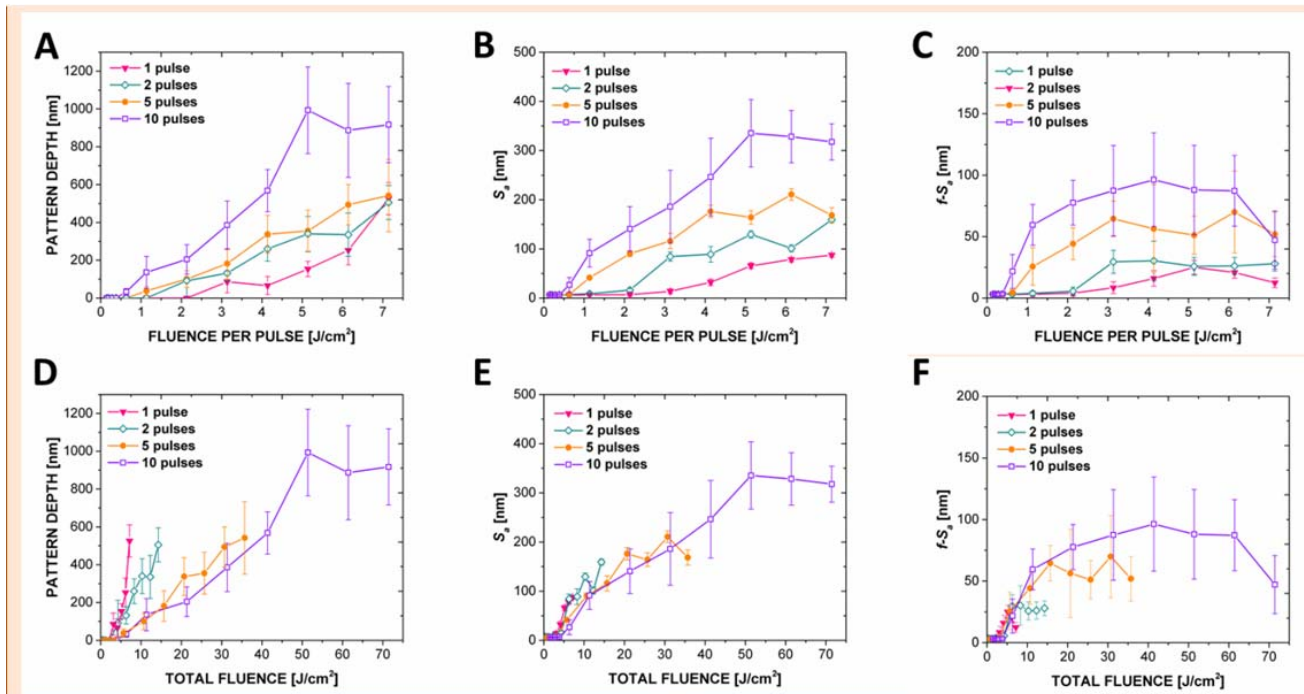

[u3]

Figure 4. Amplitude Roughness parameters of DLIP treated zirconia surfaces as a function of laser parameters. Pattern depth as a function of fluence per pulse (A) and total fluence (D), for increasing number of pulses. Average Roughness $\left(S_{a}\right)$ as a function of fluence per pulse $(B)$ and total fluence $(E)$, for increasing number of pulses. Low-pass-Filtered Average Roughness $\left(f-S_{a}\right)$ as a function of fluence per pulse $(C)$ and total fluence $(F)$, for increasing number of pulses.

It can be observed that increasing the fluence per pulse and the number of pulses allows obtaining deeper structures, after the fluence threshold has been reached (see Fig. 4A). In Figure 4D the structure depth is plotted against the total fluence delivered to the sample during the treatment. It is interesting to note that the total depth depends on the number of pulses, achieving more depth for fewer pulses even if the same total fluence is delivered to the surface. [u4] In Figure $4 \mathrm{~B}$ the plot of $S_{a}$ as a function of increasing laser fluence shows that the overall roughness of the treated surfaces also increases with both laser fluence and number of pulses. In Figure $4 \mathrm{E}$ the same quantity is plotted as a function of total fluence. Differently from pattern depth, it can be observed that different combinations of laser 
parameters delivering the same total fluence produce the same overall roughness. The abovementioned $S_{a}$ values are calculated on the non-filtered image and therefore have contributions from both the pattern and the smaller-scale roughness.

In order to deconvolute the two contributions and isolate the small-scale roughness, a Gaussian filter was applied. Figure $4 C$ plots $f-S_{a}$ as a function of increasing laser fluence per pulse and shows that after the threshold fluence has been reached the small scale roughness stays almost constant with increasing fluence but it increases with increasing number of pulses. Similarly, plotting $f-S_{a}$ versus the total fluence (Fig. 4F), shows that the small scale roughness does not depend on the total fluence but only on the number of pulses.

In Figure 5 SEM images of the surface treated with different combinations of fluence and number of pulses are presented.

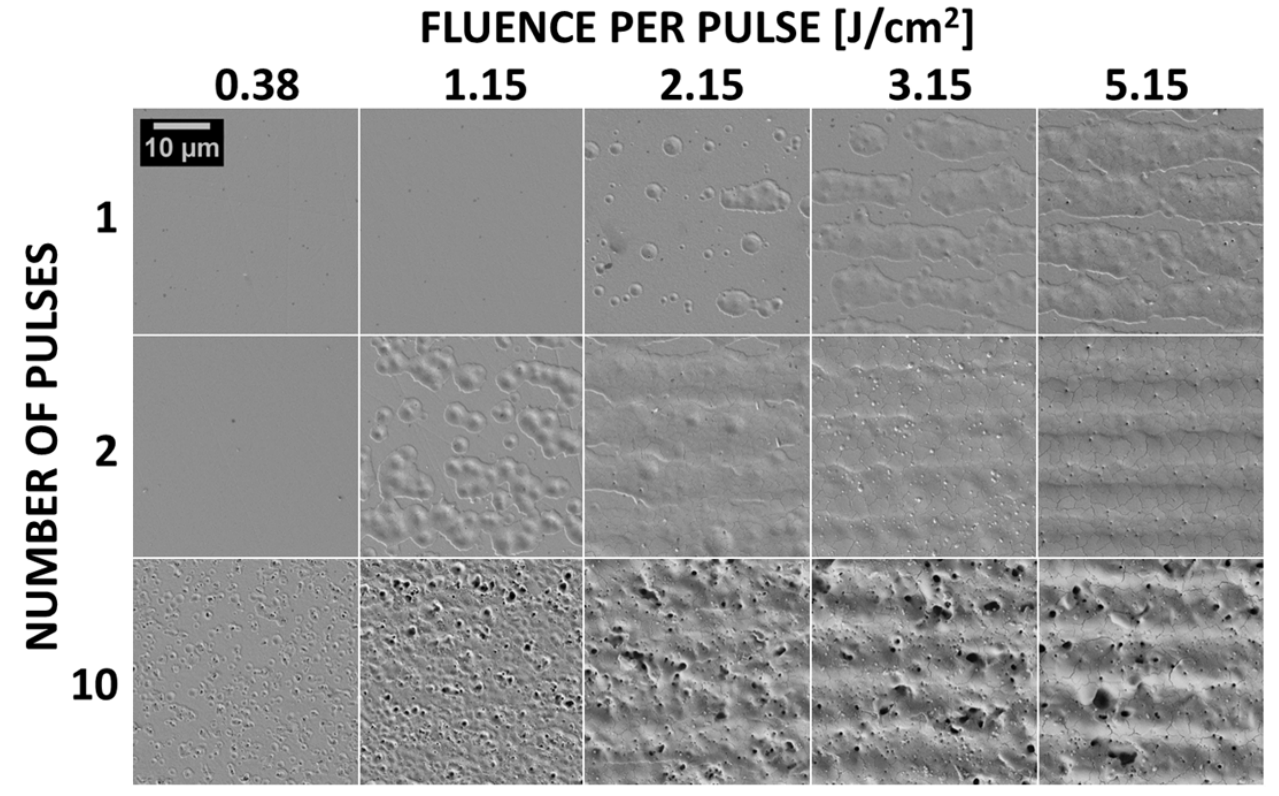

Figure 5. SEM images of DLIP treated zirconia surfaces with different laser parameters combinations. Pattern evolution as a function of laser parameters: with increasing fluence, from left to right, and with increasing number of pulses, from top to bottom. 
The series of samples treated with one pulse shows the pattern formation mechanism with increasing fluence (Fig. 5). Close to the threshold fluence $\left(2.15 \mathrm{~J} / \mathrm{cm}^{2}\right)$, circular spots of modified material appear aligned along lines corresponding to fluence maxima. Increasing the fluence (between $3.15 \mathrm{~J} / \mathrm{cm}^{2}$ and $5.15 \mathrm{~J} / \mathrm{cm}^{2}$ ), the molten spots widen and coalesce along fluence maxima lines, forming continuous bands alternated to non-modified material. For treatments with two and ten pulses, the pattern lines develop completely at lower fluence per pulse. Treating the surface with two pulses allow formation of clear lines while with ten pulses the pattern becomes blurred and lines lose definition due to the formation of open porosity on the surface.

A Fourier spatial analysis was performed to confirm previous observations.
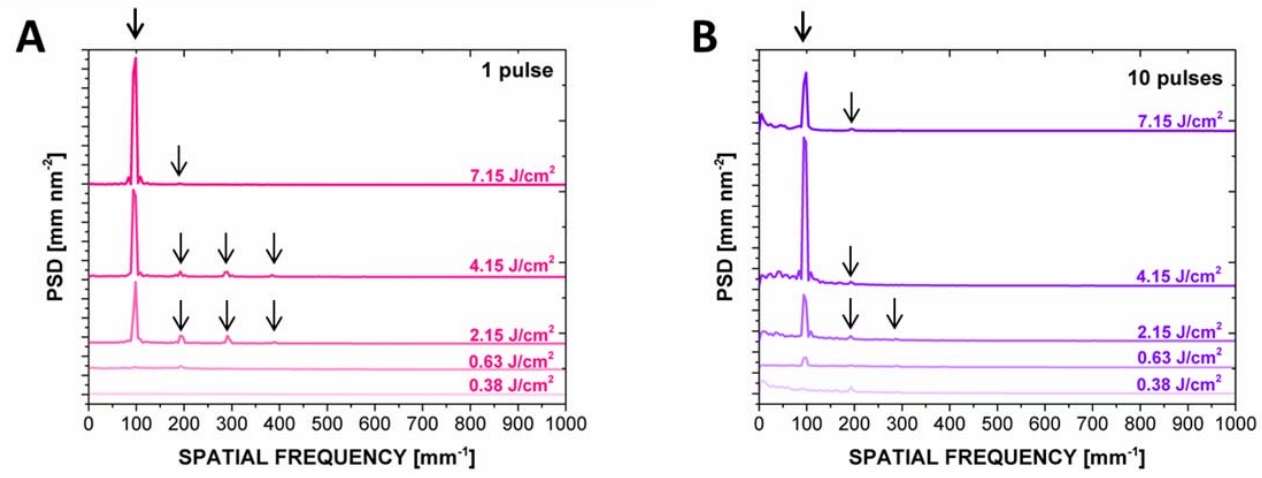

Figure 6. Fourier spatial analysis of 3Y-TZP surfaces treated with DLIP with different laser parameter combinations. In the graphs, PSD is calculated on WLI profiles orthogonal to pattern lines and is plotted as a function of spatial frequencies. Series of samples treated with one pulse (A) and ten pulses (B) with increasing fluence. Arrows highlights the main peak and the secondary frequencies.

In Figure 6A the Power Spectral Density (PSD) as a function of spatial frequencies for increasing fluence for treatments with one pulse is presented. The main peak observed at $100 \mathrm{~mm}^{-1}$ (bold black arrow) corresponds to the pattern periodicity of $10 \mu \mathrm{m}$ and appears at the threshold fluence of $2.15 \mathrm{~J} / \mathrm{cm}^{2}$. Secondary peaks at $200 \mathrm{~mm}^{-1}, 300 \mathrm{~mm}^{-1}$ and $400 \mathrm{~mm}^{-1}$ (black arrows) correspond to higher spatial frequencies superimposed to the main profile. The amplitude of the main peak increases with fluence while the secondary peaks decrease: the higher spatial frequencies disappear for higher fluence 
treatments, resulting in a quasi-sinusoidal profile. These secondary frequencies come from the partially developed pattern due to the liquid rims that have not joined (see SEM images in Figure 5 for one pulse treatment). In Figure $6 \mathrm{~B}$ the trend of PSD as a function of fluence for treatments with ten pulses is presented. They differ from those of one pulse mainly because of the noise at low frequencies (comprised between 0 and $250 \mathrm{~mm}^{-1}$ ). The main peak at $100 \mathrm{~mm}^{-1}$ appears above the threshold fluence of $0.63 \mathrm{~J} / \mathrm{cm}^{2}$. Its amplitude increases with fluence until a maximum is reached but then the noise contribution overcomes its relative amplitude. The noise increment with pulse number is compatible with the increment in $f$-S (see Fig. $4 \mathrm{C}$ ): both are due to the formation of pores on the surface.

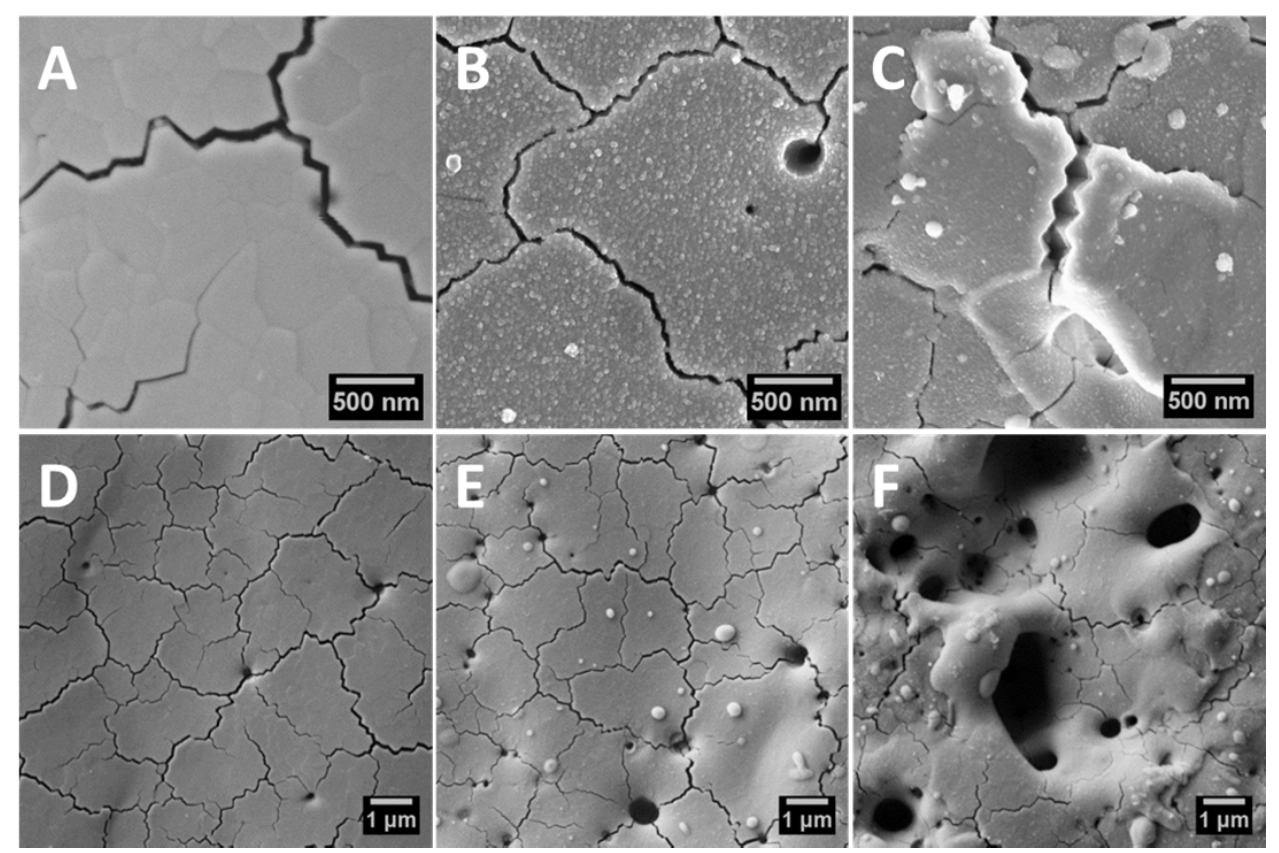

Figure 7. SEM images of DLIP treated zirconia surfaces with different laser parameter combinations. The samples were treated at maximum fluence per pulse $\left(7.15 \mathrm{~J} / \mathrm{cm}^{2}\right)$ and with two $(A, D)$, five $(B, E)$ and ten (C, F) pulses, respectively.

Closer inspection of treated surfaces (see Fig. 7A, B, C), where interconnected intergranular cracks are evident, reveals that the resolidified material is cracked for all the laser conditions used. 
Figure 7D, E, F illustrate the different aspect and size of pores depending on the pulse number. For treatments with two pulses (Fig. 7D) few circular pores appear on the surface (with a diameter of around 200nm), while for treatments with five and ten pulses (Fig. 7E, F) pores become larger, not spherical and with different sizes between $200 \mathrm{~nm}$ and $1.7 \mu \mathrm{m}$. Bigger pores are always surrounded by rims, often layered, and drops (with a diameter of $390 \pm 120 \mathrm{~nm}$ ) are scattered all over the surrounding surface (Fig. 7E, F). On surfaces treated with combinations of five pulses and fluence per pulse above $2.15 \mathrm{~J} / \mathrm{cm}^{2}$ and with combinations of ten pulses for all fluences, nano-droplets cover homogeneously the surface (see Fig. 7B, C).

\section{DISCUSSION}

DLIP exploits the inhomogeneous fluence distribution created by interference to engrave a pattern on the surface of the treated material. Due to the relatively long pulse duration (in the order of nanoseconds), patterning will be obtained by thermal mechanisms, implying melting and evaporation of the material accompanied by liquid flow [24,37]. However, some contribution of photo-physical mechanisms may not be discarded [23], [25].

The traces of molten material (Fig. 5) indicate that the local temperature of the surface has reached the melting temperature of the material $\left(\approx 2700^{\circ} \mathrm{C}\right)[38]$, as it has been previously reported for zirconia ceramics treated with ns-pulsed laser in the UV range [28,39]. The periodical distribution of fluence on the surface of the material results in an analogous temperature distribution: the highest temperature is reached at fluence maximum and it decreases moving towards the zero fluence position $[26,29,37]$. The inhomogeneity in temperature distribution produces a surface tension gradient inside the liquid which results in mass flow due to thermocapillarity [40].Therefore, the molten material flows from the highest temperature zone (lower surface tension) towards the lower temperature zone (higher surface tension) [41-43]. As fluence is increased, molten pools deepen and widen since a larger volume is exposed to a fluence superior to the threshold value, as it can be expected. This results in deeper structures and the 
maximum is reached when liquid rims from two adjacent stripes join forming the complete line pattern, as depicted in Fig. 8 for one pulse treatment.

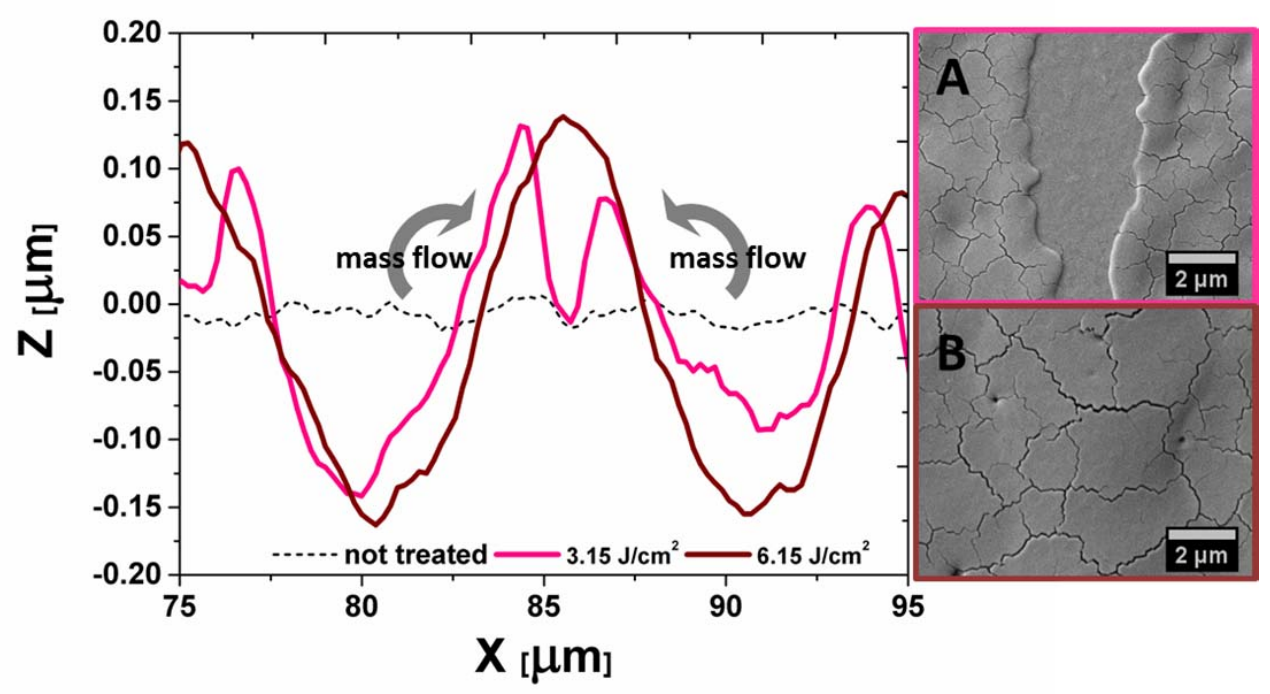

Figure 8. Pattern profiles taken from WLI images (orthogonal to pattern lines) and corresponding SEM image of the surface: comparison between non treated surface and surfaces treated with one pulse and $3.15 \mathrm{~J} / \mathrm{cm}^{2}(\mathrm{~A})$ and $6.15 \mathrm{~J} / \mathrm{cm}^{2}$ (B). Mechanism of pattern formation due to mass flow is presented in the graph. For intermediate fluences (A) the molten material of neighbour lines does not join, and for higher fluence (B) the fronts merge.

The resulting pattern profile comes as a consequence of the number of pulses and the fluence employed. In the case of one pulse treatment, as observed in the WLI (Fig. 3) and SEM images (Fig. 5), pattern formation involves melting of the material after fluence exceeds the threshold value (2.15 $\mathrm{J} / \mathrm{cm}^{2}$ ). The surface treated with intermediate fluence (between $2.15 \mathrm{~J} / \mathrm{cm}^{2}$ and $6.15 \mathrm{~J} / \mathrm{cm}^{2}$ ) results in a pattern with continuous lines of molten material alternated with unmodified material. The profile presents a depression corresponding to the molten pool alongside with two rims corresponding to the molten material flown from the liquid pool toward the colder region. The two parallel liquid fronts from adjacent pools do not join, forming a topography consisting of alternating valley and double peaks (Fig. 
$8 \mathrm{~A})$. The surface treated with higher fluence (above $6.15 \mathrm{~J} / \mathrm{cm}^{2}$ ) presents a completely developed pattern where the parallel liquid fronts have joined: the profile is almost sinusoidal, since the liquid fronts merged forming a single peak (Fig. 8B).

Treatments with two pulses show similar topography evolution (see Fig. 3 and Fig.5). However, all the process takes place at lower values of fluence since the available energy to melt the substrate material comes as the addition of the first and the subsequent pulse reaching the sample.

For five and ten pulses treatments threshold fluence further decrease and also the distribution of the first melting pools becomes more scattered and does not follow strictly fluence distribution on the surface, as a result of pulse incubation effects [44]. Material melting still occurs but the presence of open porosity on the surface suggests also bubble formation. A circular liquid rim surrounding the pores (Fig. 7F) indicates that the porosity could be attributed to exploding bubbles due to liquid-vapor instability. This kind of porosity has already been observed for different DLIP treatments on Yttria stabilized zirconia [28] but the origin of these bubbles is controversial: it can be attributed to preexisting porosity inside the bulk that is freed by material melting and can reach the surface or to bubble formation inside the bulk due to boiling or local overheating during laser-material interaction. Further increment of fluence and number of pulses provokes the formation of smaller droplets on the surface (Fig. 7B, C). These nano-scale droplets may be attributed to vapor or plasma condensation, two phases that form when the laser absorbed energy is increased, as it has already been reported in Yttria stabilized zirconia during ns-laser treatments [39].

Pattern formation is always associated to material melting and the resolidified material is always cracked (Fig. 5). Cracks are intergranular, interconnected and form a continuous net over the treated surface. Right after material resolidification the temperature difference between the surface and the bulk is very high, generating steep temperature gradients which results in surface thermal shock. Intergranular cracking has already been observed for ultraviolet nanosecond laser treatments on $3 Y$ TZP $[39,45,46]$, but not for DLIP[28]. 


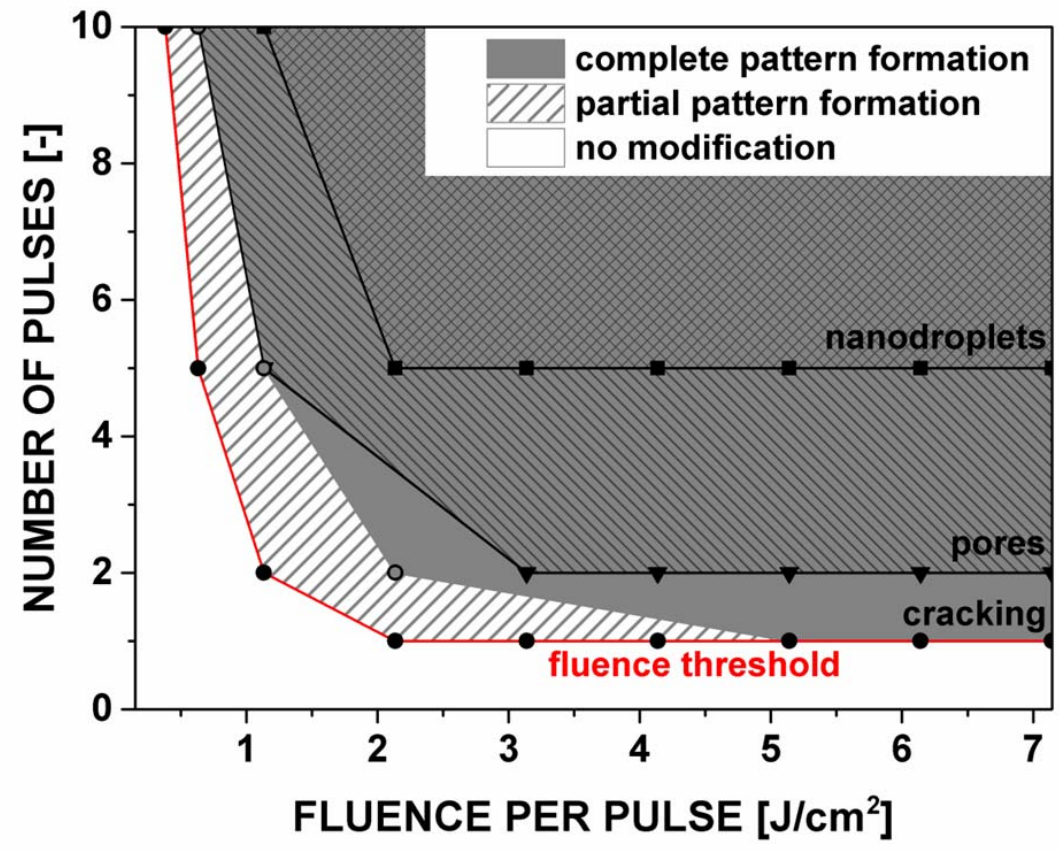

Figure 9. Parametric processability map of 3Y-TZP treated with DLIP: pattern formation and associated collateral damage as a function of laser fluence and number of pulses. The red line connects the experimental points of fluence threshold: above these energies, patterning can be achieved.

All these observations have been combined into a processability map (see Fig.9), in an attempt to schematize the influence of laser parameters on surface finish. This map has been divided into five different areas: for fluences below the threshold no surface modification is produced while above these values the pattern can develop completely or partially. Pattern formation involves material melting and it is always accompanied by cracking, in the tested conditions. For treatments with five and ten pulses complete pattern formation is always associated to pores appearance, while for one and two pulses patterning without developing bubbles at low fluences can be achieved. For treatments with more than five pulses at high fluences smaller droplets form.

In summary, the overall roughness results from the superposition of the pattern and the smaller-size features of defects produced (like cracks and pores). Laser parameters combination influence both and 
this is reflected in the dependence of roughness parameters from fluence and number of pulses. The two adjustable laser parameters have distinct but not separable effect on the resulting morphology and care should be taken on the choice of the desired combination.

Before selecting laser parameters combination it is important not only to consider the desired depth of the pattern or the roughness desired, but also its quality. In order to obtain deeper patterns both the fluence and the number of pulses can be increased. However, as Fourier Analysis and SEM observations have shown, this leads to a loss in the definition of the pattern. Regular lines can be achieved with treatments with low number of pulses (one or two) but are limited to a given depth (500 nm for 7.15 $\left.\mathrm{J} / \mathrm{cm}^{2}\right)$. On the other hand, higher depth can be achieved $(1 \mu \mathrm{m}$ at $7.15 \mathrm{~J} / \mathrm{cm} 2$ with ten pulses), but the quality of the pattern is reduced. Since small scale roughness is sensitive to pulse number but not fluence it would be possible to create patterns with different depth and overall roughness but similar small-scale roughness simply by tuning laser fluence. This would be interesting from a biomedical point of view since the biological response is sensitive to topography at various scales[47].

However, DLIP on 3Y-TZP is not free of collateral damage: microcracking is always present on the treated surface due to the high thermal gradients. For high number of pulses also open porosity is left after the treatment.[F5][u6]

\section{CONCLUSION}

The surface of 3Y-TZP has been treated with DLIP producing periodic line-like pattern in a relatively fast and inexpensive way. Varying the optical setup it was possible to produce patterns with periodicities in the range between 3 and $15 \mu \mathrm{m}$.

The role of laser parameters (fluence and number of pulses) on the produced topography has been investigated for patterns with $10 \mu \mathrm{m}$ periodicity. From the obtained results, we can conclude that:

1. Tuning laser parameters allows varying independently pattern depth, roughness and surface finish, generating a wide set of geometries. 
2. The best conditions in terms of quality of the produced pattern and minimum material damage is obtained for low number of pulses (one or two) with high laser fluence $\left(7.15 \mathrm{~J} / \mathrm{cm}^{2}\right)$.

3. Deeper patterns (up to $1 \mu \mathrm{m}$ ) can be achieved with high number of pulses (five or ten) and high fluence $\left(7.15 \mathrm{~J} / \mathrm{cm}^{2}\right)$. For these conditions pattern neatness is reduced due to the introduction of porosity on the surface;

4. Smaller scale roughness appears due to porosity formation and redeposition of volatilized material. This roughness depends on surface defects and is sensitive to pulse number more than laser fluence.

5. Surface damage (intergranular cracking, open porosity and nano-droplets formation) can be produced, depending on laser parameters.

More research is needed to understand if all the produced defects may be detrimental to the mechanical performance and the long-term reliability of the material.

\section{AKNOWLEDGEMENTS[u7]}

The authors would like to acknowledge the European Commission funding under ...[u8] and the support of the Ministry of Economy and Competitiveness (MINECO) of Spain (project ref. MAT2014-60720-R). The authors would also like to thank Dr. Trifon Trifonov for his help during SEM sessions, Quentin Flamant for his valuable advice on roughness analysis and Dr. Carsten Gachot, Björn Lechtaler and Leander Reinert for their help with the laser setup.

\section{REFERENCES}

[1] Powers JM, Sakaguchi RL. Craig's Restorative Dental Materials. 12th ed. Elsevier; 2006.

[2] Kohal R-J, Att W, Bächle M, Butz F. Ceramic abutments and ceramic oral implants. An update. Periodontol 2000 2008;47:224-43.

[3] Hisbergues M, Vendeville S, Vendeville P. Zirconia: Established facts and perspectives for a biomaterial in dental implantology. J Biomed Mater Res B Appl Biomater 2009;88:519-29. doi:10.1002/jbm.b.31147. 
[4] Manicone PF, Rossi lommetti P, Raffaelli L. An overview of zirconia ceramics: basic properties and clinical applications. J Dent 2007;35:819-26. doi:10.1016/j.jdent.2007.07.008.

[5] Denry I, Kelly J. State of the art of zirconia for dental applications. Dent Mater 2008;24:299-307. doi:10.1016/j.dental.2007.05.007.

[6] Quirynen M, Bollen CM. The influence of surface roughness and surface-free energy on supraand subgingival plaque formation in man. A review of the literature. J Clin Periodontol 1995;22:1-14.

[7] Treccani L, Yvonne Klein T, Meder F, Pardun K, Rezwan K. Functionalized ceramics for biomedical, biotechnological and environmental applications. Acta Biomater 2013;9:7115-50. doi:10.1016/j.actbio.2013.03.036.

[8] Laranjeira MS, Carvalho Â, Pelaez-Vargas A, Hansford D, Ferraz MP, Coimbra S, et al. Modulation of human dermal microvascular endothelial cell and human gingival fibroblast behavior by micropatterned silica coating surfaces for zirconia dental implant applications. Sci Technol Adv Mater 2014;15:025001. doi:10.1088/1468-6996/15/2/025001.

[9] Anil S, Anand PS, Alghamdi H, Jansen J a. Dental Implant Surface Enhancement and Osseointegration. Implant Dent - A Rapidly Evol Pract 2011:83-108. doi:10.5772/16475.

[10] Usumez A, Hamdemirci N, Koroglu BY, Simsek I, Parlar O, Sari T. Bond strength of resin cement to zirconia ceramic with different surface treatments. Lasers Med Sci 2013;28:259-66. doi:10.1007/s10103-012-1136-x.

[11] Bärsch N, Barcikowski S, Baier K. Ultrafast-Laser-Processed Zirconia and its Adhesion to Dental Cement. J Laser Micro/Nanoengineering 2008;3:78-83. doi:10.2961/jlmn.2008.02.0004.

[12] Liu D, Matinlinna JP, Tsoi JKH, Pow EHN, Miyazaki T, Shibata Y, et al. A new modified laser pretreatment for porcelain zirconia bonding. Dent Mater 2013;29:559-65. doi:10.1016/j.dental.2013.03.002.

[13] Chintapalli RK, Marro FG, Jimenez-Piqué E, Anglada M. Phase transformation and subsurface damage in 3Y-TZP after sandblasting. Dent Mater 2013;29:566-72. doi:10.1016/j.dental.2013.03.005.

[14] Muñoz-Tabares JA, Jimenez-Piqué E, Reyes-Gasga J, Anglada M. Microstructural changes in ground 3Y-TZP and their effect on mechanical properties. Acta Mater 2011;59:6670-83. doi:10.1016/j.actamat.2011.07.024.

[15] Flamant Q, García Marro F, Roa Rovira JJ, Anglada M. Hydrofluoric acid etching of dental zirconia. Part 1: etching mechanism and surface characterization. J Eur Ceram Soc 2015;36:121-34. doi:10.1016/j.jeurceramsoc.2015.09.021.

[16] Calvo-Guirado JL, Ramos-Oltra ML, Negri B, Delgado-Ruíz RA, Ramirez-Fernández PR, MateSánchez JE, et al. Osseointegration of zirconia dental implants modified by femtosecond laser vs. Zirconia implants in healed bone: A histomorphometric study in dogs with three-month followup. J Osseointegration 2013;5:39-44.

[17] Calvo-Guirado JL, Aguilar Salvatierra a, Gargallo-Albiol J, Delgado-Ruiz R a, Mate Sanchez JE, Satorres-Nieto M. Zirconia with laser-modified microgrooved surface vs. titanium implants 
covered with melatonin stimulates bone formation. Experimental study in tibia rabbits. Clin Oral Implants Res 2014:1421-9. doi:10.1111/clr.12472.

[18] Holthaus MG, Treccani L, Rezwan K. Comparison of micropatterning methods for ceramic surfaces. J Eur Ceram Soc 2011;31:2809-17. doi:10.1016/j.jeurceramsoc.2011.07.020.

[19] Samant AN, Dahotre NB. Laser machining of structural ceramics-A review. J Eur Ceram Soc 2009;29:969-93. doi:10.1016/j.jeurceramsoc.2008.11.010.

[20] Lawrence JR., Dowding C., Griffiths JB., editors. Laser surface engineering: processes \& applications. Elsevier. 2014.

[21] Mücklich F, Lasagni AF, Daniel C. Laser Interference Metallurgy - using interference as a tool for micro / nano structuring. Int J Mat Res 2006;97:1337-44.

[22] Engleman P, Dahotre N, Kurella A. The application of laser-induced multi-scale surface texturing. Jom 2005:46-50.

[23] Hans M, Gachot C, Müller F, Mücklich F. Direct laser interference structuring as a tool to gradually tune the wetting response of titanium and polyimide surfaces. Adv Eng Mater 2009;11:795-800. doi:10.1002/adem.200900115.

[24] Hans M, Müller F, Grandthyll S, Hüfner S, Mücklich F. Anisotropic wetting of copper alloys induced by one-step laser micro-patterning. Appl Surf Sci 2012;263:416-22.

[25] Gachot C, Catrin R, Lasagni AF, Schmid U, Mücklich F. Comparative study of grain sizes and orientation in microstructured $\mathrm{Au}, \mathrm{Pt}$ and $\mathrm{W}$ thin films designed by laser interference metallurgy. Appl Surf Sci 2009;255:5626-32. doi:10.1016/j.apsusc.2008.10.032.

[26] Daniel C, Mücklich F, Liu Z. Periodical micro-nano-structuring of metallic surfaces by interfering laser beams. Appl Surf Sci 2003;208-209:317-21. doi:10.1016/S0169-4332(02)01381-8.

[27] Berger J, Grosse Holthaus M, Pistillo N, Roch T, Rezwan K, Lasagni AF. Ultraviolet laser interference patterning of hydroxyapatite surfaces. Appl Surf Sci 2011;257:3081-7. doi:10.1016/j.apsusc.2010.10.120.

[28] Daniel C, Armstrong BL, Howe JY, Dahotre N. Controlled Evolution of Morphology and Microstructure in Laser Interference-Structured Zirconia. J Am Ceram Soc 2008;91:2138-42. doi:10.1111/j.1551-2916.2008.02449.x.

[29] Lasagni A, Seyler M, Holzapfel C, Maier WF, Mücklich F. Periodical Gratings in Mixed-Oxide Films by Laser-Interference Irradiation. Adv Mater 2005;17:2228-32. doi:10.1002/adma.200402074.

[30] Li P, Bakowsky U, Yu F, Loehbach C, Muecklich F, Lehr C-M. Laser ablation patterning by interference induces directional cell growth. IEEE Trans Nanobioscience 2003;2:138-45.

[31] Mathews S, Hans M, Mücklich F, Solioz M. Contact killing of bacteria on copper is suppressed if bacterial-metal contact is prevented and is induced on iron by copper ions. Appl Environ Microbiol 2013;79:2605-11. doi:10.1128/AEM.03608-12. 
[32] Daniel C, Drummond J, Giordano RA. Improving Flexural Strength of Dental Restorative Ceramics Using Laser Interference Direct Structuring. J Am Ceram Soc 2008;91:3455-7. doi:10.1111/j.15512916.2008.02642.x.

[33] Wennerberg A, Albrektsson T. On implant surfaces: a review of current knowledge and opinions. Int J Oral Maxillofac Implants 2009;25:63-74.

[34] Muñoz-Tabares JA, Jiménez-Piqué E, Anglada M. Subsurface evaluation of hydrothermal degradation of zirconia. Acta Mater 2011;59:473-84. doi:10.1016/j.actamat.2010.09.047.

[35] EN ISO 13356:2013 2013.

[36] Bäuerle DW. Laser processing and chemistry. 4th ed. Springer; 2011.

[37] Lasagni AF, Mücklich F. Study of the multilayer metallic films topography modified by laser interference irradiation. Appl Surf Sci 2005;240:214-21. doi:10.1016/j.apsusc.2004.06.143.

[38] Chevalier J, Gremillard L, Virkar A V., Clarke DR. The Tetragonal-Monoclinic Transformation in Zirconia: Lessons Learned and Future Trends. J Am Ceram Soc 2009;92:1901-20. doi:10.1111/j.1551-2916.2009.03278.x.

[39] Heiroth S, Koch J, Lippert T, Wokaun a., Günther D, Garrelie F, et al. Laser ablation characteristics of yttria-doped zirconia in the nanosecond and femtosecond regimes. J Appl Phys 2010;107:014908. doi:10.1063/1.3275868.

[40] Bennett TD, Krajnovich DJ, Grigoropoulos CP, Baumgart P, Tam a C. Marangoni mechanism in pulsed laser texturing of magnetic disk substrates. J Heat Transf Asme 1997;119:589-96. doi:10.1115/1.2824146.

[41] Berg S. Marangoni-driven spreading along liquid-liquid interfaces. Phys Fluids 2009;21. doi:10.1063/1.3086039.

[42] Karbalaei A, Kumar R, Cho H. Thermocapillarity in Microfluidics-A Review. Micromachines 2016;7:13. doi:10.3390/mi7010013.

[43] Saiz E, Tomsia AP. Atomic dynamics and Marangoni films during liquid-metal spreading. Nat Mater 2004;3:903-9. doi:10.1038/nmat1252.

[44] Paltauf G, Dyer PE. Photomechanical processes and effects in ablation. Chem Rev 2003;103:487518. doi:10.1021/cr010436c.

[45] Stübinger S, Homann F, Etter C, Miskiewicz M, Wieland M, Sader R. Effect of Er : YAG, CO2 and diode laser irradiation on surface properties of zirconia endosseous dental implants. LASERS Surg Med 2008;40:223-8. doi:10.1002/lsm.20614.

[46] Ihlemann J, Scholl A, Schmidt H, Wolff-Rottke B. Nanosecond and femtosecond excimer-laser ablation of oxide ceramics. Appl Phys A 1995;60.

[47] Ito Y. Surface micropatterning to regulate cell functions. Biomaterials 1999;20:2333-42. 